\title{
Observed and predicted premature mortality in Australia due to non- communicable diseases: a population- based study examining progress towards the WHO 25X25 goal
}

\author{
Alison Wijnen ${ }^{1}$, Karen Bishop ${ }^{1}$, Grace Joshy ${ }^{1}$, Yuehan Zhang ${ }^{1}$, Emily Banks ${ }^{1,2}$ and Ellie Paige ${ }^{1 *}$ (D)
}

\begin{abstract}
Background: The World Health Organization's (WHO) 25X25 goal aims for a 25\% relative reduction in premature death due to four non-communicable diseases (NCD4) - cancer, cardiovascular disease, chronic respiratory diseases and diabetes - by 2025 compared to 2010. This study aimed to quantify the premature mortality in the Australian population due to NCD4, quantify the variation in mortality rates by age and sex, predict the premature mortality due to NCD4 in 2025 and evaluate the progress towards the WHO 25X25 goal.

Methods: A population-based study using cause-specific mortality data of all deaths which occurred in Australia from 2010 to 2016 and registered up to 2017, for adults aged 30-69 years, was conducted. Age-specific and agestandardised mortality rates (ASMR) and probability of death for NCD4 were calculated for each year. ASMRs in 2016 were calculated for men and women. Deaths and the probability of death in 2025 were predicted using Poisson regression based on data from 2006 to 2016. To assess the progress against the WHO 25X25 goal, the relative reduction in the probability of death from NCD4 conditions in 2025 compared to 2010 was calculated.

Results: ASMRs for NCD4 decreased from 2010 to 2016, except for diabetes which increased on average by 2.5\% per year. Across sociodemographic factors, ASMRs were highest in males and increased with age. The projected probability of premature death in 2025 was 7.36\%, equivalent to a relative reduction of $25.16 \%$ compared to 2010 levels.
\end{abstract}

\footnotetext{
* Correspondence: ellie.paige@anu.edu.au

${ }^{1}$ National Centre for Epidemiology and Population Health, Australian National University, Canberra, Australia

Full list of author information is available at the end of the article
}

C C The Author(s). 2022 Open Access This article is licensed under a Creative Commons Attribution 4.0 International License, which permits use, sharing, adaptation, distribution and reproduction in any medium or format, as long as you give appropriate credit to the original author(s) and the source, provide a link to the Creative Commons licence, and indicate if changes were made. The images or other third party material in this article are included in the article's Creative Commons licence, unless indicated otherwise in a credit line to the material. If material is not included in the article's Creative Commons licence and your intended use is not permitted by statutory regulation or exceeds the permitted use, you will need to obtain permission directly from the copyright holder. To view a copy of this licence, visit http://creativecommons.org/licenses/by/4.0/ The Creative Commons Public Domain Dedication waiver (http://creativecommons.org/publicdomain/zero/1.0/) applies to the data made available in this article, unless otherwise stated in a credit line to the data. 
Conclusions: Premature mortality due to cancer, cardiovascular disease, respiratory diseases and diabetes declined in Australia from 2010 to 2016. This trend is consistent across age groups and by sex, and higher mortality rates were observed in males and at older ages. Nationally, if the current trends continue, we estimate that Australia will achieve a 25.16\% relative reduction in premature deaths due to NCD4 in 2025 compared to 2010, signifying substantial progress towards the WHO 25X25 goal. Concerted efforts will need to continue to meet the $25 \times 25$ goal, especially in the context of the COVID-19 pandemic.

Keywords: Non-communicable diseases, Cancer, Cardiovascular disease, Chronic respiratory diseases, Diabetes, WHO 25X25 goal, Premature mortality, Sociodemographic factors

\section{Background}

Non-communicable diseases (NCDs) cause around 15 million deaths annually worldwide, in people aged $30-69$ years, most of which are preventable with changes to modifiable risk factors [1]. NCDs are responsible for over $70 \%$ of deaths globally and $89 \%$ of deaths in Australia [2]. In Australia, NCDs comprise the majority of the burden of disease with cancer being the largest contributor, followed by cardiovascular diseases (CVD), with respiratory diseases and endocrine diseases, including diabetes, being the 6th and 9th largest contributors, respectively, in 2015 [3].

In 2013, member states of the World Health Organization (WHO), including Australia, agreed to adopt a global framework for monitoring progress towards decreasing mortality from NCDs [4]. The NCD monitoring framework has nine voluntary targets for 2025 , including a target to reduce premature mortality from four NCDs (hereafter referred to as NCD4) by $25 \%$ compared to 2010 levels: known as the 25X25 goal. Progress towards the WHO $25 \mathrm{X} 25$ goal is measured by a relative reduction in the probability of death from four major NCDs-cancer, CVD, chronic respiratory diseases and diabetes-in people aged 30-69 years inclusive [5].

A number of studies have been published considering progress towards the $25 \mathrm{X} 25$ goal and the related 30 by 2030 sustainable development goal (SDG) which aims for a $30 \%$ relative reduction in the probability of premature death by 2030 compared to 2015 levels. These indicate that WHO methodology and modelling can be used to measure premature mortality and evaluate the progress towards the goal. A Swedish study showed that a $25 \%$ relative reduction in premature mortality due to NCDs had been achieved in the period 1991 to 2006 [6]. They concluded that further reductions to achieve the 25X25 goal may be challenging in Sweden and other countries with similarly low mortality rates [6]. A UK study modelled future population, mortality and population exposure to risk factors and showed that reducing levels of obesity would have the greatest reduction on expected mortality in 2025 [7]. A study conducted in China examined the progress towards the 2030 SDG [8]. They found that the target would not be met due to expected trends in cancer and diabetes mortality [8]. The NCD Countdown Collaborators took a similar approach to examine the 2030 SDG and found the lowest probability of premature death in 2015 was in highincome countries including Australia. Their modelling indicated that for Australia to meet the 2030 goal, a faster rate of decline would be needed in several disease groups including chronic respiratory diseases [9].

A recent $\mathrm{WHO}$ report on international progress towards the NCD monitoring framework goals showed that in 2016 the probability of NCD premature death for Australia was low in comparison with international standards, at $9.0 \%$. This represents a $9.2 \%$ relative reduction compared to the probability of $9.9 \%$ reported in 2010 [10]. NCD Countdown Collaborators [11] have modelled the probability of premature death up to 2030 . However, there are no published projections on the expected relative decrease in NCD deaths in Australia by 2025 specifically or investigation of the relative contribution of the individual NCDs towards meeting the WHO 25X25 goal. There is also limited data on how mortality due to NCD4 varies in Australia by age and sex. Such data are important not only for identifying whether Australia is likely to meet the WHO 25X25 goal based on current trends, but for identifying possible points to intervene to further reduce mortality from NCD4 across the population.

The aims of this study are to (1) quantify premature mortality in the Australian population from 2010 to 2016 that is due to the four major NCDs: cancer, CVD, chronic respiratory diseases and diabetes, separately and combined; (2) quantify variation in premature NCD4 mortality rates by age and sex; (3) predict expected premature mortality for the NCD4 separately and combined in 2025; and (4) compare progress against the WHO $25 \mathrm{X} 25$ goal target probability of dying from one of the NCD4 conditions.

\section{Methods}

\section{Study population}

This study is based on adults resident in Australia aged 30-69 years in 2010-2016 using data on the Estimated Resident Population for Australia [12]. 


\section{Data sources}

Deaths registered in Australia from 2006 to 2016 for the study population were obtained from the Cause of Death Unit Record Files provided by the Australian Registrars of Births, Deaths and Marriages and the Coroners and the National Coronial Information System. Australian mortality data have a five-star rating (highest possible) for data quality, including coverage of death registrations, from the Global Burden of Disease study. Across all ages for the years of interest, there were only 126 records with age not stated. Several different sources of information are used to identify sex when not stated, resulting in no missing data by sex for the mortality data used in this study.

The Estimated Resident Population for Australia and projected Australian populations were obtained from the Australian Bureau of Statistics. The Australian Bureau of Statistics provides three alternate population projection scenarios, series A, B and C, for Australia [13]. Series B reflects current trends in migration, fertility and life expectancy, while series $\mathrm{A}$ and $\mathrm{C}$ predict the expected Australian populations under an increase and decrease, respectively, in trends of migration, fertility and life expectancy. Series B population projection scenarios are used in the main analysis and series $\mathrm{A}$ and $\mathrm{C}$ are used in sensitivity analyses (details below).

\section{Outcome}

The main outcome was death from one of NCD4, ascertained from the underlying cause of death in the Cause of Death Unit Record Files. The International Classification of Diseases 10, Australian Modification (ICD-10-AM) codes were used to identify deaths due to cancer (CO0C97), CVD (I00-I99), chronic respiratory diseases (J30J98) and diabetes (E10-E14) $[14,15]$.

\section{Statistical analysis}

\section{Observed deaths, death rates and probability of dying}

Measures were calculated using 5-year age and sex groups, for each NCD4 separately (CVD, cancer, chronic respiratory diseases, diabetes) and combined.
Age-standardised mortality rates (ASMRs) for cancer, CVD, chronic respiratory diseases and diabetes were calculated separately and combined for the population aged 30-69, for each year 2010-2016, using direct standardisation to the 2011 Estimated Resident Population of Australia [12]. Death records with missing age at death were excluded $(n=83)$.

The annual percentage change in ASMRs was calculated for each year in the period 2010-2016 and averaged over this period.

Using the life table method recommended for the WHO $25 \times 25$ indicator (Table 1), we calculated the annual age-specific probability of premature death in 5year age groups for people aged 30-69 years and each NCD4 condition, over the period 2010-2016 [14].

\section{Projected deaths, death rates and probability of dying}

Poisson regression with a log-linear link function was used to predict the number of NCD4 deaths in each year from 2017 to 2025 by sex and 5-year age groups. Poisson regression was used as the outcome modelled was count data (number of deaths), and log-linear link function was used to ensure that modelled decreasing trends in mortality would not fall below zero. The Poisson models included the observed annual number of deaths in each 5 -year age and sex group using all available data from 2006 to 2016 as the dependent variable, time/year as the independent variable and an offset term was included as the exposure time represented by the Estimated Resident Population in the corresponding year of projected population series B, 2017-2025. The model predicted the number of NCD4 deaths which were then used with the projected populations for Australia from 2017 to 2025 to calculate annual age-specific and age-standardised mortality rates for each NCD4 condition through to 2025 . We used series B population projections for the main analysis to predict deaths on current trends in migration, fertility and life expectancy.

The projected age-specific mortality rates were used to estimate the probability of premature death, for each year 2017 to 2025 . The target probability in 2025 was

Table 1 Life table method for calculating the unconditional probability of death

$$
{ }_{40}^{*} q_{30}=1-\prod_{x=30}^{65}\left(1-{ }_{5}^{*} q_{x}\right)
$$

$40 \mathrm{q} 30$ is the probability of dying (from an NCD4 condition) between ages 30 and 70

where ${ }_{5}^{*} q_{x}=\frac{{ }_{5}^{*} M_{x} \times 5}{1+{ }_{5}^{*} M_{x} \times 2.5}$ is the probability of death in each five-year age group and ${ }_{5}^{*} M_{x}$ is the age-specific mortality rate for each five-year age group for ages $x=30,35, \ldots, 65$. 
calculated as the probability of premature death observed in 2010, multiplied by 0.75 to estimate a $25 \%$ reduction. The percentage reduction in the projected probability of premature death was calculated for 2025 compared to 2010 , to estimate whether the target $25 \%$ reduction was met.

\section{Subgroup analysis}

To assess whether premature mortality, projected agespecific mortality rates and the probability of death from the NCD4 in 2025 varied by age or sex, age-specific rates and ASMRs in 2016 were calculated for each NCD4 condition and by sex (male and female). The projected probability of premature death in 2025 was calculated for each NCD4 separately, and combined, and by sex.

\section{Sensitivity analyses}

Two sensitivity analyses were conducted to assess the influence of key assumptions on the projection model.

First, we calculated the projected ASMRs and probability of premature death in 2025 using alternate population projection scenarios, series $\mathrm{A}$ and $\mathrm{C}$, for Australia [13], reflecting projected changes in the Australian populations under an increase and decrease, respectively, in trends of migration, fertility and life expectancy.

Second, we used two different methods accounting for multiple causes of death to address the reliance of the analysis on the use of underlying cause of death, as specified by the WHO methodology. To account for multiple causes of death, associated causes of deaths listed in the deaths data were used to identify and recalculate the number of deaths in two ways: (1) counting deaths by any mention of an NCD4 as an underlying or associated cause and (2) counting deaths by assigning equal weighting to each cause contributing to the death, except duplicate mentions and ill-defined associated causes [16]. These alternate counts of the number of deaths were used to calculate the premature ASMR and probability of premature death in 2010-2016 and the projected premature ASMR and probability of premature death 2017-2015 as in the main analysis.

\section{Validation of projection estimates for 2017}

Deaths in 2017 were not included in the main analysis due to lags in death registration, affecting about $4-7 \%$ of deaths in a given year [17].

Instead, the number of deaths estimated to occur in 2017 was estimated using the projection models. To check the validity of the projections for 2017, the observed rates for deaths registered in 2017 were compared to predicted rates for 2017 using a rate ratio. We defined a priori that a point estimate for the rate ratio for projected vs observed ASMR between 0.9 and 1.1 would be acceptable.

\section{Results}

The study population in 2016, consisted of $12,155,608$ adults resident in Australia aged 30-69 years, with $50.64 \%$ females (Table 2).

Observed deaths, death rates and probability of dying In 2016, there was a total of 158,426 deaths across the population aged 18 years and older, of which 105,128 $(66.36 \%)$ were due to NCD4. Of the population aged $30-69$ years, there were 36,327 deaths in 2016 (sex ratio of 1.63 of males to females), of which 25,082 (69.05\%) were due to all NCD4 conditions combined. Most deaths (42.94\%) were due to cancer, followed by CVD (18.42\%) (Table 2). The number of people in the population, total number of deaths and proportion of the total mortality by each NCD4 condition is provided for each year (2010-2016) in Additional file 1: Table S1.

Over the period 2010-2016, 172,522 people aged 3069 years died from all NCD4 conditions combined. The highest premature ASMRs were observed for cancer followed by CVD, chronic respiratory diseases and diabetes (Fig. 1). The premature ASMRs declined from 137.11 deaths per 100,000 in 2010 to 123.53 in 2016 for cancer (1.72\% average annual decrease), 61.16 to 53.18 for CVD (2.28\% average annual decrease), and 14.16 to 13.82 for chronic respiratory diseases $(0.31 \%$ average annual decrease), contributing to a decline of 219.46 to 198.58 deaths per 100,000 for NCD4 conditions combined (Additional file 1: Table S2). Premature ASMR due to diabetes increased from 7.03 deaths per 100,000

Table 2 Study population aged 30-69 years, deaths and proportional mortality in 2016, for cancer, cardiovascular disease (CVD), respiratory diseases and diabetes (NCD4)

\begin{tabular}{|c|c|c|c|c|c|c|c|c|c|}
\hline & \multirow[t]{2}{*}{ Population } & \multirow{2}{*}{$\begin{array}{l}\text { \% of total } \\
\text { Australian population } \\
\text { (all ages) }\end{array}$} & \multirow{2}{*}{$\begin{array}{l}\text { Number } \\
\text { of } \\
\text { deaths }\end{array}$} & \multicolumn{6}{|c|}{ Proportional mortality (\%) by underlying cause of death } \\
\hline & & & & Cancer & CVD & $\begin{array}{l}\text { Chronic respiratory } \\
\text { diseases }\end{array}$ & Diabetes & $\begin{array}{l}\text { NCD4 } \\
\text { combined }\end{array}$ & $\begin{array}{l}\text { Other } \\
\text { causes }\end{array}$ \\
\hline Persons & $12,155,608$ & 50.25 & 36,327 & 42.94 & 18.42 & 4.89 & 2.79 & 69.05 & 30.96 \\
\hline Males & $6,000,459$ & 24.81 & 22,501 & 38.64 & 21.25 & 4.39 & 2.92 & 67.20 & 32.80 \\
\hline Females & $6,155,149$ & 25.44 & 13,826 & 49.94 & 13.80 & 5.71 & 2.60 & 72.05 & 27.96 \\
\hline
\end{tabular}

Notes: The proportional mortality was calculated at the national level as the percentage of total deaths within the study population 
250
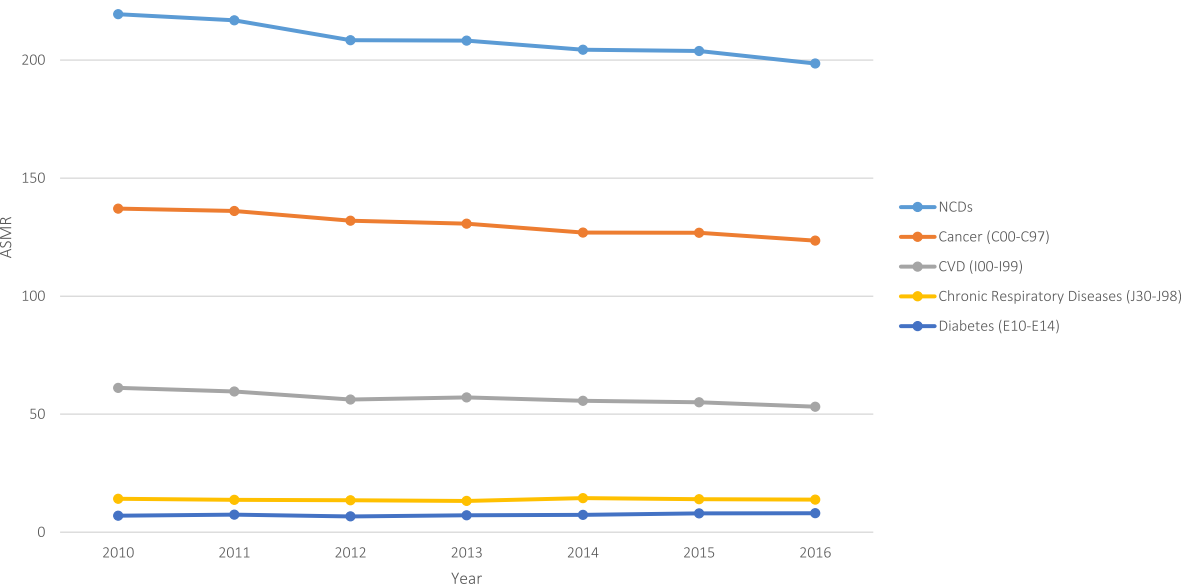

$\rightarrow$ Diabetes (E10-E14)

Fig. 1 Age-standardised premature mortality rates for cancer, cardiovascular disease, respiratory diseases and diabetes combined in adults resident in Australia aged 30-69 years from 2010 to 2016. Notes: (1) The age-standardised mortality rates (ASMR) per 100,000 population were calculated at the national level and age-standardised using the 2011 Australian Estimated Resident Population of people aged 30-69. (2) The ASMRs for cancer, cardiovascular disease (CVD), chronic respiratory diseases and diabetes combined is displayed as non-communicable diseases (NCDs)

in 2010 to 8.04 in 2016, with an average annual increase of $2.98 \%$ (Additional file 1: Table S2).

The probability of premature death from NCD4 declined from $9.84 \%$ in 2010 to $8.89 \%$ in 2016 (Table 3). This means a 30-year old living in Australia in 2016 had an $8.89 \%$ chance of dying from one of the NCD4s before reaching their 70 th birthday, if the observed mortality rates for that year persisted at each age, and death did not occur due to another cause [18].

\section{Projected deaths, death rates and probability of dying}

Projected numbers of deaths and proportional mortality for each NCD4 condition for the years 2017 to 2025 are provided in Additional file 1: Table S1. The projected

Table 3 Probability of dying aged 30-69 years in Australia, 2010-2016, from cancer, cardiovascular disease (CVD), respiratory diseases and diabetes (NCD4)

\begin{tabular}{|c|c|c|c|c|c|}
\hline \multirow[t]{2}{*}{ Year } & \multirow[t]{2}{*}{ Cancer } & \multicolumn{4}{|c|}{ Underlying cause of death } \\
\hline & & CVD & Chronic respiratory diseases & Diabetes & NCD4 combined \\
\hline \multicolumn{6}{|c|}{ Probability of premature death (\%) } \\
\hline 2010 & 6.26 & 2.82 & 0.70 & 0.33 & 9.84 \\
\hline 2011 & 6.20 & 2.73 & 0.68 & 0.36 & 9.71 \\
\hline 2012 & 6.04 & 2.58 & 0.67 & 0.32 & 9.38 \\
\hline 2013 & 5.98 & 2.62 & 0.66 & 0.34 & 9.36 \\
\hline 2014 & 5.80 & 2.55 & 0.71 & 0.35 & 9.17 \\
\hline 2015 & 5.77 & 2.52 & 0.69 & 0.37 & 9.12 \\
\hline 2016 & 5.63 & 2.43 & 0.68 & 0.38 & 8.89 \\
\hline \multicolumn{6}{|c|}{ Projected premature age-standardised mortality rate per 100,000 in 2025} \\
\hline 2025 & 104.56 & 40.21 & 13.46 & 7.73 & 164.23 \\
\hline \multicolumn{6}{|c|}{ Projected probability of premature death in 2025 (\%) } \\
\hline 2025 & 4.77 & 1.81 & 0.66 & 0.35 & 7.36 \\
\hline \multicolumn{6}{|c|}{ Relative reduction in the projected probability of death compared to $2010(\%)$} \\
\hline 2025 & - & - & - & - & 25.16 \\
\hline
\end{tabular}

Notes: (1) Rates represent deaths per 100,000, standardised to using the 2011 Australian Estimated Resident Population of people aged 30-69 years. (2) The probability of premature death due to cancer (C00-C97), cardiovascular disease (CVD) (I00-199), chronic respiratory diseases (J30-J98) and diabetes (E10-E14) combined is displayed under non-communicable diseases (NCD4) combined 
Table 4 Probability of premature death for people aged 30-69 years, in Australia, 2005 projected to 2025, from cancer, cardiovascular disease, respiratory diseases and diabetes (NCD4)

\begin{tabular}{|c|c|c|c|c|c|c|}
\hline \multirow[t]{3}{*}{ Year } & \multicolumn{6}{|c|}{ Probability of premature death due to NCD4 (\%) } \\
\hline & \multicolumn{3}{|c|}{ WHO estimate (24) } & \multicolumn{3}{|c|}{ Australian estimate } \\
\hline & Male & Female & Person & Male & Female & Person \\
\hline $2005^{\mathrm{a}} / 2006$ (2005 for WHO estimate) & 13.90 & 8.80 & 11.40 & 13.12 & 8.26 & 10.71 \\
\hline 2010 & 12.10 & 7.80 & 10.00 & 12.02 & 7.63 & 9.84 \\
\hline 2015 & 11.30 & 7.30 & 9.30 & 11.10 & 7.15 & 9.12 \\
\hline 2016 & 11.00 & 7.20 & 9.10 & 10.83 & 6.98 & 8.89 \\
\hline 2025 (target) & 9.08 & 5.85 & 7.50 & 9.02 & 5.72 & 7.38 \\
\hline 2025 (projected) & - & - & - & 8.90 & 5.90 & 7.36 \\
\hline
\end{tabular}

Notes: (1) The probability of premature death due to non-communicable diseases (NCD4) represents the probability of death in persons aged 30-69 inclusive, in Australia, due to cancer (C00-C97), cardiovascular disease (CVD) (I00-199), chronic respiratory diseases (J30-J98) and diabetes (E10-E14) combined. (2) The projected probability of premature death in 2025 was calculated using census and mortality data from 2006 to 2016 with the Australian Bureau of Statistics projected population series B which reflects the current trends in migration, fertility and life expectancy. (3) The WHO data can be viewed online [10]. (4) The probability of premature death in 2025 needed to meet the WHO 2025 target was calculated as the WHO probability of premature death in 2010 multiplied by 0.75 (to estimate a $25 \%$ reduction). (A) WHO estimate is given for 2005, and the Australian estimate was calculated for 2006

premature ASMR due to NCD4 conditions combined in 2025 was 164.23 per 100,000 (Table 3 and Additional file 1: Table S3). In 2025, the projected probability of premature death was $7.36 \%$ (Table 4 and Fig. 2) compared to $9.84 \%$ in 2010 , representing a predicted relative reduction of $25.16 \%$ by 2025 (Table 3). Model fit was good (Additional file 1: Table S4).

\section{Subgroup analysis}

Within the population aged $30-69$ years, rates of premature death increased with increasing age (Fig. 3) and premature ASMRs were higher in men compared to women for each NCD4 condition (Fig. 4). The same cause-specific patterns were observed by age and sex: the highest rates were for cancer followed by CVD, chronic respiratory diseases and diabetes. The greatest difference in ASMRs between males and females was for CVD (Fig. 4). The projected probability of premature death from NCD4 conditions in 2025 was higher in males than in females $(8.90 \%$ vs. $5.90 \%$; Additional file 2: Fig. S1).

\section{Sensitivity analyses}

Using the different projected population scenarios, the probability and rate of premature mortality were slightly lower for series A (increased migration, fertility and life expectancy) and slightly higher for series $\mathrm{C}$ (decreased migration, fertility and life expectancy) compared with the results based on series B (current trends) (Fig. 2). However, this variation was small with all premature ASMRs for series $\mathrm{A}$ and $\mathrm{C}$ being within 1-unit death per 100,000 from series B, and the probability of premature death for series $A$ and $C$ being within $0.01 \%$ points from series B. The probability of premature death in 2025 was 7.35\% for series A (increased migration, fertility and life expectancy) and $7.38 \%$ for series $C$ (decreased migration, fertility and life expectancy) (Additional file 1: Table S5).

Patterns of results differed slightly from the main analysis when methods accounting for multiple causes of death were used (Additional file 2: Figs. S2-S4). Compared to the main results, the probability of premature death was higher using the method of any mention $(11.32 \%)$ and lower using the equal weighting method (6.42\%). This resulted in a relative reduction in the probability of death in 2025 compared to 2010 levels of $23.46 \%$ and $26.80 \%$, respectively (Additional file 1 : Table S6).

\section{Projection validation}

There was little difference $(1.37 \%$ deviation and a rate ratio of 1.01) between the observed and projected ASMRs for NCD4 conditions combined for 2017 (Additional file 1: Table S7).

\section{Discussion}

Our study shows that if current trends in the mortality rate declines of NCD4 conditions continue, Australia can expect to see a reduction in the probability of premature death due to cancer, CVD, chronic respiratory diseases and diabetes, of around $25.16 \%$ by 2025 . This suggests that meeting the WHO $25 \mathrm{X} 25$ goal is possible if actions to reduce NCDs continue.

Our large-scale study using whole-of-population data showed a decline in premature mortality in the Australian population due to cancer, CVD, chronic respiratory diseases and diabetes combined over the period 20102016, with age-standardised mortality rates declining by an average of $1.64 \%$ annually. Compared to 2010 levels, the probability of premature death is projected to reduce by around $25.16 \%$ in 2025 . 


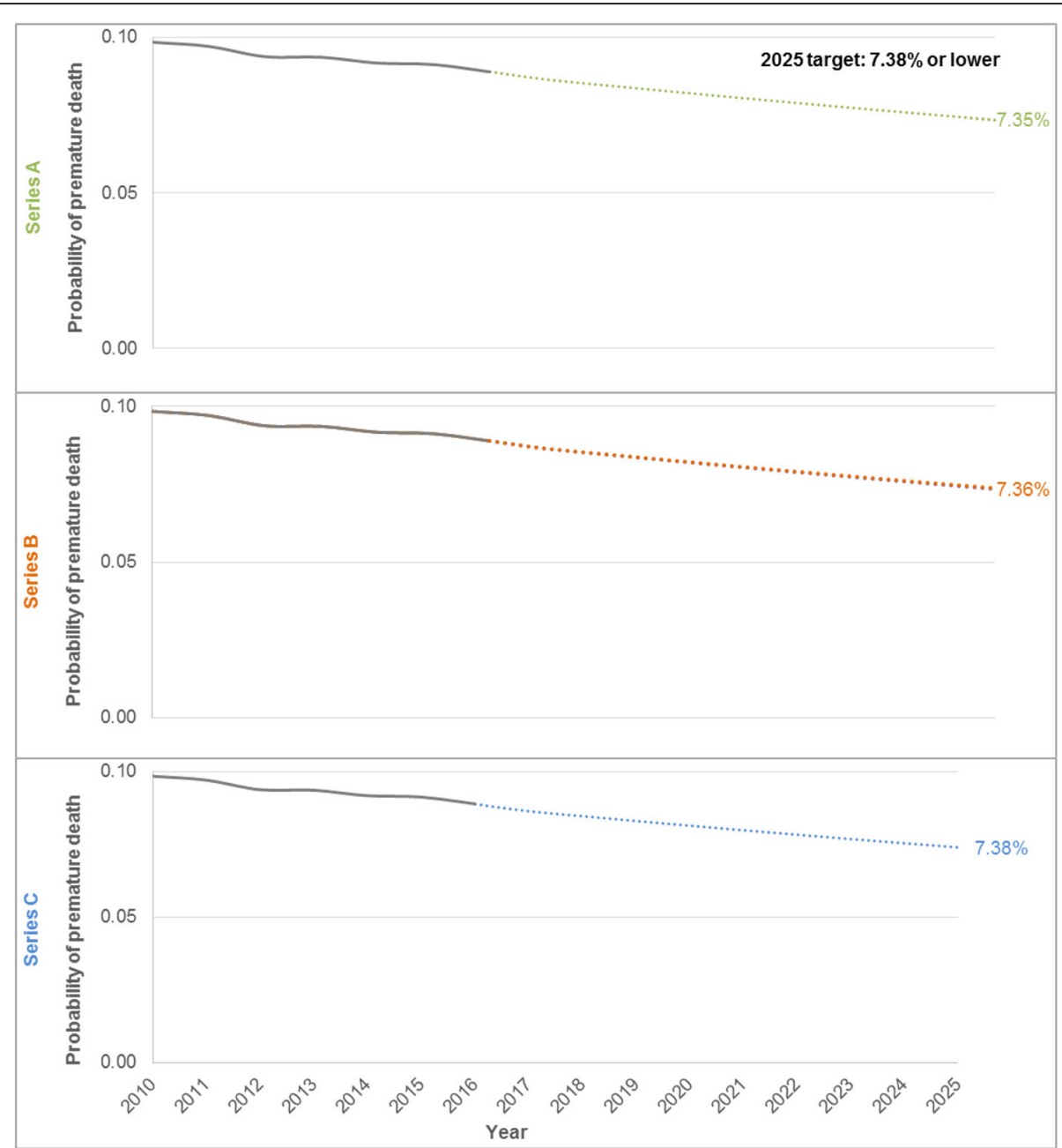

Fig. 2 Probability of premature death due to cancer, cardiovascular disease, respiratory diseases and diabetes combined (NCD4), in Australia 2010-2025, by projected populations A, B, and C. Notes: (1) Solid lines indicate observed probability and non-solid/dashed lines indicate projected probability. (2) The projected probability of premature death in 2017-2025 was calculated using the data from 2006 to 2016 with the Australian Bureau of Statistics projected population series B which reflects the current trends in migration, fertility and life expectancy (main analysis, shown in orange). Sensitivity analyses using the alternative Australian Bureau of Statistics projected population series are shown in green (series A, reflecting an increase in migration, fertility and life expectancy) and purple (series C, showing a decrease in trends in migration, fertility and life expectancy). (3) Premature death refers to deaths in people between the exact ages of 30 and 69 years. (4) Deaths were included for NCD4 conditions which were identified using ICD-10 codes: cancer (C00-C97), cardiovascular disease (CVD) (I00-I99), chronic respiratory diseases (J30-J98) and diabetes (E10-E14)

Modelling by the NCD Countdown Collaborators relates to 2030 SDG targets, rather than 2025 ones and estimated decreases of $>15$ and $\leq 20 \%$ in NCD4 conditions in Australia by 2030 compared to 2015 levels [9]. Australia has low premature NCD mortality by international standards, and this may contribute to a slower rate of decline in mortality. We expanded on previous analyses, by not only predicting premature mortality rates for NCDs by 2025 but also examining predicted rates for NCD4 conditions separately and by age and sex to provide information on points to target to further lower mortality rates. Taken together, our current analyses and those of the NCD Countdown Collaborators show that, if past trends continue, Australia might just meet the WHO 25X25 goal but is unlikely to meet the SDG goal of a $30 \%$ reduction by 2030 unless further declines in mortality are achieved.

The probability of premature death calculated for 2016 in the data (female $6.9 \%$, male $10.8 \%$ ) is very close to estimates from WHO and NCD Countdown Collaborators (female $7.1 \%$, males $11 \%$ ) [10, 11]. The observed decline in premature mortality due to NCD4 conditions combined over time is consistent with findings of a previous study that showed a reduction in the burden of disease from 2003 to 2015 [3] with age-standardised fatal burden (years of life lost) rates for cancer, CVD and 


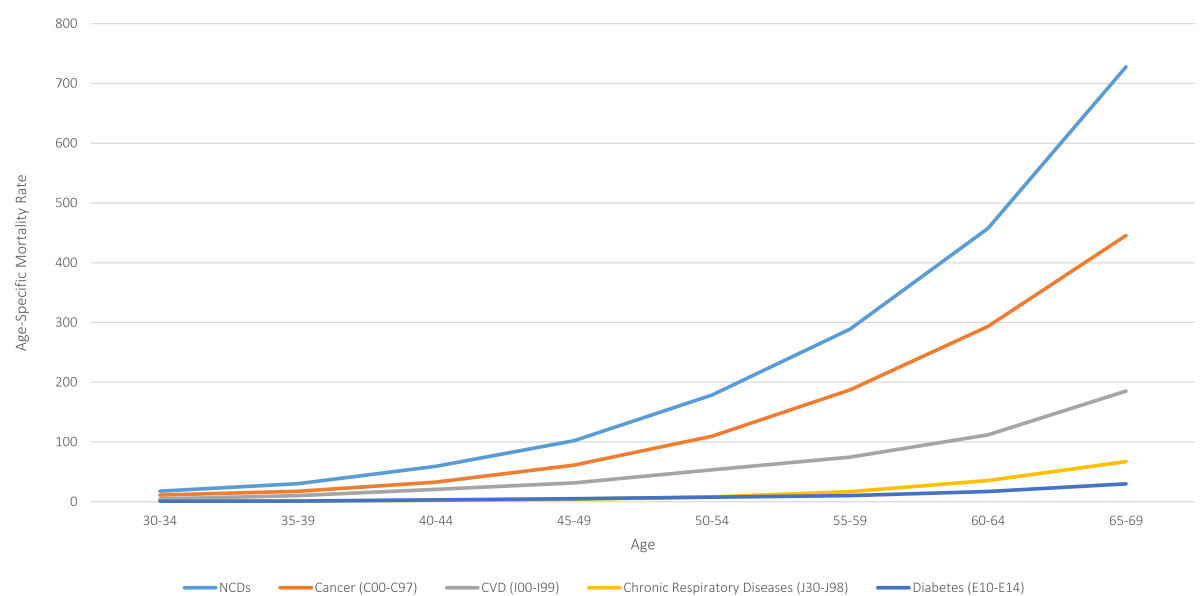

Fig. 3 Age-specific mortality rates for cancer, cardiovascular disease (CVD), respiratory diseases and diabetes separately and combined in Australia, in 2016, by underlying cause of death across 5-year age groups. Notes: (1) The age-specific mortality rate per 100,000 population, for cancer, cardiovascular disease (CVD), chronic respiratory diseases and diabetes combined, is displayed as deaths caused by non-communicable diseases (NCDs).

respiratory diseases declining by a total of $6.1 \%, 11.3 \%$ and $0.5 \%$, respectively. These findings [3] are consistent with estimates in our study of average annual decreases in ASMR of $1.72 \%$ for cancer, $2.28 \%$ for CVD and $0.32 \%$ for chronic respiratory diseases, from 2010 to 2016. While the previous study [3] reported no change in agestandardised years of life lost rate for diabetes over time, we observed a small increase in premature ASMR for diabetes. This is likely due to the changes in mortality coding associated with diabetes that were introduced in 2014 [19].

The subgroup analysis showed that while premature mortality rates declined in all groups, they were highest in males and older people. This is consistent with previously observed trends in Australia which have shown that males have had a persistently higher ASMR for all cancers combined compared to females from 1982 to 2016 and an increased fatal burden of chronic

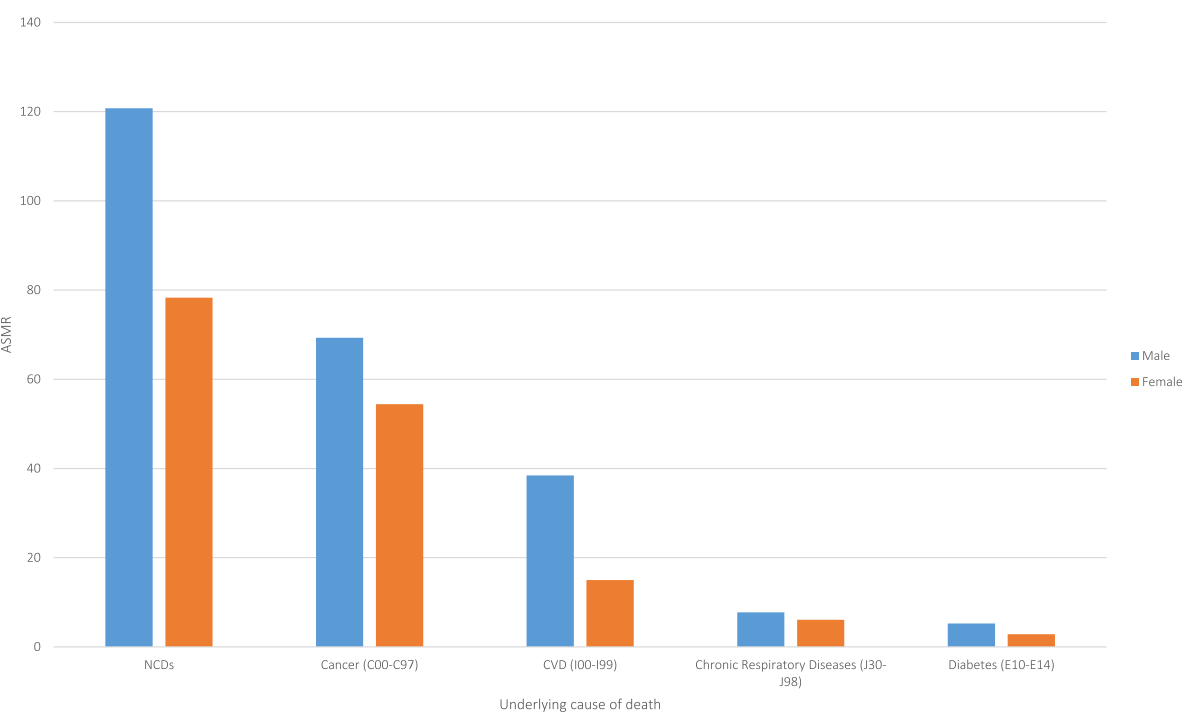

Fig. 4 Age-standardised premature mortality rates by sex, for cancer, cardiovascular disease (CVD), respiratory diseases and diabetes in adults resident in Australia aged 30-69years, in 2016. Notes: (1) The age-standardised mortality rates (ASMR) per 100,000 population were calculated at the national level and were age-standardised using the 2011 Australian Estimated Resident Population of people aged 30-69. (2) The agestandardised mortality rate for cancer, cardiovascular disease (CVD), chronic respiratory diseases and diabetes combined is displayed as deaths caused by non-communicable diseases (NCDs) 
respiratory diseases in 2011, despite experiencing a similar total burden as females [20,21]. Higher NCD mortality with increasing age was observed in our study and is well established in previous work including excess mortality rates of cancer from 1982 to 2003 and CVD mortality rates from 1980 to 2015 [22, 23].

This study is the first to predict the likelihood with which Australia will meet the WHO 25X25 goal, generating projections for the probability of premature mortality in 2025 in the context of this goal. The strengths of this study include the use of high-quality nationallevel data and the robustness of the results to different sensitivity analyses.

The large size and fine level of data in the Cause of Death Unit Record File allowed fine agestandardisation to compare rates over time. Future projections are sensitive to the data used to generate the projections as well as changes to population structures over time. To improve the stability of the projections, we used all available past data (20062016) to estimate future projections. We also undertook sensitivity analyses estimating the probability of death under two alternative projected population structures representing changes to fertility, migration and life expectancy. The projected probabilities of premature mortality were similar under these different scenarios, showing the results were robust to the assumptions of these models.

However, it is possible that the current COVID-19 pandemic will substantially impact trends in population growth, particularly migration, as Australia closed its borders to non-citizens between March 2020 and November 2021 [24]. This impact on the population structure may be considerable, given the role of net overseas migration as the largest source of population growth in Australia contributing $60.2 \%$ of population growth in 2019 [25]. Although we attempted to test for this using the alternative projected population structures provided by the Australian Bureau of statistics, these may not fully capture future changes resulting from the pandemic. Although mortality due to COVID-19 has been low in Australia so far, there may be increases in the number of preventable deaths over the next few years. For example, an increase in cancer deaths may result from reduced and delayed screening, diagnostic and treatment service utilisation which has been observed to coincide with the COVID-19 pandemic in Australia during 2020 [26]. Similar changes may be expected for other conditions such as CVD which rely on engagement with primary care for prevention and treatment.

The WHO 25X25 goal is based on reducing the probability of premature death from NCDs where these conditions are recorded as the underlying cause of death. However, death can often be attributed to multiple causes. In addition to examining the underlying cause of death in the main analysis, we used two alternative measures to account for each NCD4 condition's contribution to death in the sensitivity analyses. The first which considered any mention of NCD4 conditions resulted in an increase in the estimated ASMRs, an increase in the probability of premature death during 2010-2016 and a decrease in the relative reduction of the probability of death by 2025 compared to what was observed in the main analysis. This was expected as capturing the prevalence at the death of all conditions allows for double counting of deaths, thereby inflating the numerator. We also used a multiple cause weighting approach where each cause contributing to each death is given an equal weighting relative to the total number of causes in the record. Using the multiple cause weighting approach, we projected around a $26 \%$ reduction in premature mortality from the NCD4 in 2025 compared to 2010.

In the analysis, several assumptions underpin the calculation of the probability of premature death and the projection model. Both use 5-year age groupings assuming that the subgroup trend is representative of each age contained within it.

Mortality analyses rely on the quality cause of death data. Australia has high coverage and applies international standard for coding causes and selecting underlying the cause of death. However, the Cause of Death Unit Record File is not immune from errors in certification of cause of death (e.g. by a doctor or coroner) that may arise from misdiagnosis and misreporting the role of disease in the causal sequence resulting in death [27].

To reduce premature mortality due to NCD4 conditions, for both the WHO 25X25 goal and the 2030 SDG goal, several studies have noted that countryspecific action and targeting, based on recent trends in mortality, would be most effective $[6,9,28]$. Our study gives an overview of recent trends in premature mortality in Australia and identified areas where gains could be made to facilitate progress towards the WHO 25X25 goal. Overall, our results show that premature mortality rates were higher for males and older people (particularly from 60 to 69 years), with the greatest burden being due to cancer. Given the shared risk factors between NCDs and already low mortality rates in Australia, the biggest gains are likely to be made by targeting interventions to reduce common risk factors across the population, for example, in reducing smoking, and in targeting preventive interventions and treatments to high-risk groups by increasing screening and heath checks. Evaluating and comparing the expected public health impacts of these strategies is needed to further inform health policy. 


\section{Conclusion}

Premature mortality due to cancer, cardiovascular disease, respiratory diseases and diabetes has declined in Australia from 2010 to 2016. If past national trends continue, we predict relative reductions in the probability of premature mortality in Australia from all NCD4 conditions combined of around $25.16 \%$ in 2025 compared to 2010 levels. These findings highlight the importance of continuing and enhancing evidence-based policies and programmes targeting NCDs in Australia. This includes those targeting major and shared risk factors as well as improvements in coverage of disease screening and preventive treatments.

\section{Abbreviations}

ABS: Australian Bureau of Statistics; AlHW: Australian Institute of Health and Welfare; ASMR: Age-standardised mortality rate; CVD: Cardiovascular disease; NCD4: Cancer, cardiovascular disease, respiratory diseases and diabetes; NCDs: Non-communicable diseases; SDG: Sustainable Development Goal; WHO: World Health Organization

\section{Supplementary Information}

The online version contains supplementary material available at https://doi. org/10.1186/s12916-022-02253-z.

Additional file 1: Table S1. Study population, number of deaths, and proportional mortality from NCD4 from 2010 to 2025. Table S2. Agestandardised rate and percentage change in rate of NCD4 mortality. Table S3. Projected age-standardised premature mortality rates from NCD4. Table S4. Model coefficients and Pearson's goodness of fit test for Poisson regression models. Table S5. Sensitivity analysis using population series A, B and C. Table S6. Sensitivity analysis using multiple cause of death methods. Table S7. Validation of projection model for NCD4 mortality rates in 2017.

Additional file 2: Figure S1. Probability of premature death due to NCD4 in 2010-2025. Figure S2. Age-standardised premature mortality rates for NCD4 from 2010 to 2016, using multiple cause of death method equal weighting. Figure S3. Age-standardised premature mortality rates for NCD4 from 2010 to 2016, using multiple cause of death method any mention method. Figure S4. Probability of premature death due to NCD4 from 2010 to 2025, using multiple cause of death methods.

\section{Acknowledgements}

The authors thank the Registrars of Births, Deaths and Marriages; the Coroners and the National Coronial Information System for enabling Cause of Death Unit Record File data to be used for this research.

\section{Authors' contributions}

$E P$ and $E B$ conceived the study. AW, EP, KB and GJ designed the analyses. $A W$ analysed and interpreted the data and wrote the original manuscript with input from EP and KB. ZY analysed the data for the revised manuscript. $E P$ revised the manuscript following peer review. All authors read and approved the final manuscript.

\section{Funding}

This project was supported by a Project Grant from the National Health and Medical Research Council of Australia (reference 1163214). Emily Banks is supported by the NHMRC (reference 1136128). Ellie Paige is supported by a Postdoctoral Fellowship (reference: 102131) from the National Heart Foundation of Australia.

\section{Availability of data and materials}

The population datasets generated and/or analysed during the current study are available in the Australian Bureau of Statistics repository, http://stat.data.
abs.gov.au/Index.aspx, https://www.abs.gov.au/AUSSTATS/abs@.nsf/ DetailsPage/3222.02017\%20(base)\%20-\%202066?OpenDocument.

The death data that support the findings of this study are available from the Registrars of Births, Deaths and Marriages and the Coroners and the National Coronial Information System, but restrictions apply to the availability of these data, which were used under licence for the current study, and so are not publicly available. Data are however available from the authors upon reasonable request and with permission of the Registrars of Births, Deaths and Marriages and the Coroners and the National Coronial Information System.

\section{Declarations}

\section{Ethics approval and consent to participate}

Ethics approval for this research was provided by the Australian National University HREC (2019/022). The National Cause of Death Unit Record Files (CoDURF) used in this study are de-identified data provided by the Queensland Registry of Births, Deaths and Marriages, which is the Australian Coordinating Registry for unit-record level CoDURF. The CoDURF provided does not contain identifiers such as name, address or other data fields that in isolation can identify an individual. Informed consent is not applicable for this study.

\section{Consent for publication}

Not applicable.

\section{Competing interests}

The authors declare that they have no competing interests.

\section{Author details}

${ }^{1}$ National Centre for Epidemiology and Population Health, Australian National University, Canberra, Australia. ${ }^{2}$ The Sax Institute, Sydney, Australia.

Received: 15 September 2021 Accepted: 12 January 2022

Published online: 10 February 2022

References

1. World Health Organisation. Noncommunicable diseases progress monitor 2017. Geneva: WHO; 2017.

2. World Health Organisation. Noncommunicable diseases progress monitor 2020. Geneva: WHO; 2020.

3. Australian Institute of Health and Welfare. Australian Burden of Disease Study: impact and causes of illness and death in Australia 2015. Canberra: AlHW; 2019.

4. NCD global monitoring framework [https://www.who.int/nmh/global_ monitoring_framework/en/]. Accessed 2020.

5. World Health Organisation. Global action plan for the prevention and control of noncommunicable diseases 2013-2020. Geneva: WHO; 2013.

6. Santosa A, Rocklov J, Hogberg U, Byass P. Achieving a $25 \%$ reduction in premature non-communicable disease mortality: the Swedish population as a cohort study. BMC Med. 2015;13(1):65. https://doi.org/10.1186/s12916-0150313-8.

7. Cobiac $\amalg$, Scarborough P. Translating the WHO $25 \times 25$ goals into a UK context: the PROMISE modelling study. BMJ Open. 2017;7(4):e012805. https://doi.org/10.1136/bmjopen-2016-012805.

8. Li Y, Zeng X, Liu J, Liu Y, Liu S, Yin P, et al. Can China achieve a one-third reduction in premature mortality from non-communicable diseases by 2030? BMC Med. 2017;15(1):132. https://doi.org/10.1186/s12916-017-0894-5.

9. N. C. D. Countdown collaborators. NCD Countdown 2030: pathways to achieving Sustainable Development Goal target 3.4. Lancet. 2020; 396(10255):918-934.

10. Probability of dying between exact ages 30 and 70 from any of cardiovascular disease, cancer, diabetes, or chronic respiratory (\%) [https:// www.who.int/data/gho/data/indicators/indicator-details/GHO/probability-ofdying-between-exact-ages-30-and-70-from-any-of-cardiovascular-disease-ca ncer-diabetes-or-chronic-respiratory-(-)]. Accessed 2020.

11. N. C. D. Countdown collaborators. NCD Countdown 2030: worldwide trends in non-communicable disease mortality and progress towards Sustainable Development Goal target 3.4. Lancet. 2018;392(10152):1072-88. https://doi. org/10.1016/S0140-6736(18)31992-5. 
12. Australian Bureau of Statistics: ERP by SA2 (ASGS 2016), age and sex, 2001 Onwards [https://explore.data.abs.gov.au/vis?fs[0] = ABS\%20TopicS\%2C0\%7CPEOPLE\%23PEOPLE\%23\&pg=10\&fc= ABS\%20Topics\&df[ds]=ABS_ABS_TOPICS\&df[id]=ABS_ERP_ASGS\&df[ag] $=$ ABS\&df[vs] $=1.0 .0 \& p d=2016 \% 2 C \& d q=3 . .5 T E . . A \& l y[c \mid]=A G E \& l y[r w]=A S G S$ 2011]. Accessed 2020.

13. Australian Bureau of Statistics: Population projections, Australia, 2017 (base)2066 [https://www.abs.gov.au/AUSSTATS/abs@.nsf/mf/3222.0]. Accessed 2020.

14. World Health Organization. Noncommunicable Diseases Global Monitoring Framework: indicator definitions and specifications. Geneva: WHO; 2014

15. World Health Organization. International statistical classification of diseases and related health problems - 10th revision. Geneva: WHO; 2016.

16. Moreno-Betancur M, Sadaoui H, Piffaretti C, Rey G. Survival analysis with multiple causes of death: extending the competing risks model. Epidemiology. 2017;28(1):12-9. https://doi.org/10.1097/EDE. 0000000000000531.

17. Australian Bureau of Statistics. Cause of death, Australia, 2017. Canberra: ABS; 2019.

18. World Health Organization. Global status report on noncommunicable diseases 2014. Geneva: WHO; 2014.

19. Australian Bureau of Statistics. Cause of death, Australia, 2013; technical note 1 ABS implementation of Iris software: understanding coding and process improvements. Canberra: ABS; 2016.

20. Australian Institute of Health and Welfare. The burden of chronic respiratory conditions in Australia: a detailed analysis of the Australian Burden of Disease Study 2011. Canberra: AlHW; 2017.

21. Australian Institute of Health and Welfare. Cancer in Australia 2019. Canberra: AlHW; 2019.

22. Dasgupta P, Cramb S, Kou K, Yu XQ, Baade PD. Temporal trends in net and crude probability of death from cancer and other causes in the Australian population, 1984-2013. Cancer Epidemiol. 2019;62:101568. https://doi.org/1 0.1016/j.canep.2019.101568

23. Australian Institute of Health and Welfare. Trends in cardiovascular deaths. Canberra: AlHW; 2017.

24. Murphy K, Karp M. Australian government moves to close borders as new coronavirus cases continue to rise. The Guardian: In; 2020.

25. Australian Bureau of Statistics. Australian demographic statistics, Dec 2019. Canberra: ABS; 2020.

26. Cancer Australia. Review of the impact of COVID-19 on medical services and procedures in Australia utilising MBS data: skin, breast and colorectal cancers, and telehealth services. Surry Hills, NSW: Cancer Australia; 2020.

27. Harriss LR, Ajani AE, Hunt D, Shaw J, Chambers B, Dewey H, et al. Accuracy of national mortality codes in identifying adjudicated cardiovascular deaths. Aust N Z J Public Health. 2011;35(5):466-76. https://doi.org/10.1111/j.17536405.2011.00739.x

28. Norheim OF, Jha P, Admasu K, Godal T, Hum RJ, Kruk ME, et al. Avoiding $40 \%$ of the premature deaths in each country, 2010-30: review of national mortality trends to help quantify the UN sustainable development goal for health. Lancet. 2015;385(9964):239-52. https://doi.org/10.1016/S0140-673 6(14)61591-9.

\section{Publisher's Note}

Springer Nature remains neutral with regard to jurisdictional claims in published maps and institutional affiliations.

Ready to submit your research? Choose BMC and benefit from:

- fast, convenient online submission

- thorough peer review by experienced researchers in your field

- rapid publication on acceptance

- support for research data, including large and complex data types

- gold Open Access which fosters wider collaboration and increased citations

- maximum visibility for your research: over $100 \mathrm{M}$ website views per year

At $\mathrm{BMC}$, research is always in progress.

Learn more biomedcentral.com/submissions 